\title{
ALDH Activity Correlates with Metastatic Potential in Primary Sarcomas of Bone
}

\author{
Nicholas Greco1,2, Trevor Schott1,2, Xiaodong Mu1,2, Adam Rothenberg1,2, Clifford Voigt1,2, \\ Richard L. McGough III1,3, Mark Goodman1,3, Johnny Huard 1,2,3, Kurt R. Weiss 1,2,3 \\ ${ }^{1}$ Department of Orthopaedic Surgery, University of Pittsburgh Medical Center, Pittsburgh, USA \\ ${ }^{2}$ Cancer Stem Cell Laboratory, University of Pittsburgh School of Medicine, Pittsburgh, USA \\ ${ }^{3}$ University of Pittsburgh Cancer Institute, University of Pittsburgh School of Medicine, Pittsburgh, USA \\ Email: greconj2@upmc.edu
}

Received 22 January 2014; revised 20 February 2014; accepted 27 February 2014

Copyright (C) 2014 by authors and Scientific Research Publishing Inc.

This work is licensed under the Creative Commons Attribution International License (CC BY).

http://creativecommons.org/licenses/by/4.0/

(c) (i) Open Access

\begin{abstract}
Osteosarcoma (OS), chondrosarcoma (CSA), and Ewings sarcoma (ES) are the most common primary malignancies of bone, and are rare diseases. As with all sarcomas, the prognosis of these diseases ultimately depends on the presence of metastatic disease. Survival is therefore closely linked with the biology and metastatic potential of a particular bone tumor's cells. Here we describe a significant correlation of aldehyde dehydrogenase (ALDH) activity and the presence/absence of distant metastases in ten consecutive cases of human bone sarcomas. Additionally, cultured human CSA cells, which are historically chemo- and radio-resistant, may be sensitive to the ALDH inhibitor, disulfiram. While it is premature to draw broad conclusions from such a small series, the importance of ALDH activity and inhibition in the metastatic potential of primary bone sarcomas should be investigated further.
\end{abstract}

\section{Keywords}

Osteosarcoma, Chondrosarcoma, Ewings Sarcoma, Aldehyde Dehydrogenase, Metastases

\section{Introduction}

Osteosarcoma (OS), chondrosarcoma (CSA) and Ewings sarcoma (ES) are the three most common primary malignancies of bone [1]-[6]. Most examples of "bone cancer" treated by musculoskeletal oncologists are cases wherein cancer has metastasized to the bony skeleton. Examples of primary tumors that metastasize to bone include epithelial neoplasia such as breast, prostate, renal, thyroid, and lung carcinomas [7] [8]. In contrast, primary bone sarcomas are rare diseases of mesenchymal origin that arise within the skeleton itself. As with all 
sarcomas, the prognosis of primary bone sarcoma is ultimately determined by the presence or absence of systemic metastatic disease [9]-[12]. The most common site for sarcoma metastasis is to the lung, but other sites such as bone and lymph node also occur [13]-[18].

Modern treatment strategies for OS and ES include neoadjuvant chemotherapy, wide resection of the tumor, and post-operative chemotherapy. Unfortunately, CSA is resistant to both cytotoxic chemotherapy and external beam radiotherapy, and thus is primarily a surgical disease [19] [20]. Regardless of the histologic subtype, the metastatic potential of bone sarcoma cells dramatically impacts patient survival, as cure is much less likely in the setting of systemic disease. While advances in chemotherapy and surgery have improved local control and patient functionality, there have not been significant advances in the survival of patients with bone sarcomas in over two decades [13] [21] [22]. Clearly, a more complete understanding of the biology of bone sarcoma metastases is required if prognoses are to improve.

Aldehyde dehydrogenase (ALDH) is a tetrameric enzyme that oxidizes aldehydes to carboxylic acids and enables cells to resist oxidative stress. ALDH has been implicated as a cancer stem cell marker. Cells with high ALDH levels have demonstrated enhanced tumorigenicity in multiple cancer cell types. In clinical studies, high ALDH activity predicted poor survival in breast and ovarian cancer [23]-[29]. We observed that highly metastatic murine OS cells displayed greater resistance to oxidative stress than less metastatic murine OS cells when challenged with $\mathrm{H}_{2} \mathrm{O}_{2}$, and hypothesized that diminished ALDH activity in the less metastatic cells might explain this difference. Indeed, we demonstrated and published that ALDH expression and activity are significantly greater in murine OS cells with high metastatic potential than in less metastatic murine OS cells. ALDH-high cells were also more invasive than ALDH-low cells through a semisolid matrigel matrix. Finally, we treated OS cells with the ALDH inhibitor, disulfiram, and observed that disulfiram effectively reduced ALDH activity and altered metastatic OS cell morphology, resulting in fewer filopodia and greater uniformity of shape [15] [16]. These data suggested that ALDH is important to OS metastatic biology, and its inhibition in vitro caused diminished metastatic potential.

We wished to understand the importance of ALDH in the metastatic potential of human bone sarcomas. In order to accomplish this, the ALDH activity of cultured human bone sarcomas cells was assayed and compared with the metastatic histories of the patients from whom they were derived. We also treated primary bone sarcoma cells with disulfiram and doxorubicin (a cytotoxic agent commonly used in the treatment of bone sarcomas), and evaluated their effects on human bone sarcoma cells in vitro.

\section{Materials and Methods}

\subsection{UPMC Musculoskeletal Oncology Research Registry and Tissue Bank}

The University of Pittsburgh Medical Center (UPMC) Musculoskeletal Oncology Research Registry and Tissue Bank is an Institutional Review Board-approved data collection system wherein signed written informed consent from each participant allows us to collect the clinical data of patients with benign and malignant bone tumors prospectively from the time of diagnosis throughout the entire course of care. Additionally, patients' tumor tissue may be harvested as a reagent for laboratory study at the time of biopsy or surgery. We accrued ten consecutive patients with primary bone tumors whose cells had been harvested at the time of biopsy or surgery between October, 2011 and April, 2013. Their demographic data, histologic diagnosis, and metastatic history were evaluated.

\subsection{Establishing Bone Sarcoma Cell Lines}

Tumor tissue from patients was washed with Dulbecco's Phosphate-Buffered Saline (DPBS), finely minced, and enzymatically digested at $37^{\circ} \mathrm{C}$ in $0.2 \%$ collagenase-type XI (Sigma-Aldrich) for one hour. The cells were then incubated at $37^{\circ} \mathrm{C}$ in dispase (2.4 U/ml in HBSS, Invitrogen) for 45 minutes. Cells were then washed with DPBS and centrifuged (2500 rpm, 5 minutes) to obtain a cell pellet, which was then resuspended in proliferation medium (PM-DMEM supplemented with 10\% fetal bovine serum (FBS) and 1\% penicillin-streptomycin (all from Invitrogen)). The dissociated cells in PM were passed through a $100 \mu \mathrm{m}$ filter, and inoculated into plastic tissue culture flasks. Cells were maintained at $37^{\circ} \mathrm{C}, 5 \% \mathrm{CO}_{2}$, in a humidified incubator and PM was changed every 3 days.

\subsection{ALDH Assays via Fluorescence-Activated Cell Sorting (FACS) Analysis of ALDH Activity}

The Aldelfluor Kit (STEMCELL Technologies) was used to determine the enzymatic activity of ALDH in cul- 
tured bone sarcoma cells. Human bone sarcoma cells were trypsinized, washed with DPBS, and counted using a hemocytometer. Cells were then resuspended in Aldefluor buffer at a concentration of $1 \times 10^{6} \mathrm{cells} / \mathrm{mL}$. Aldefluor buffer contains an $\mathrm{ABC}$ transport inhibitor that prevents efflux of the Aldefluor dye. Cells were then incubated at $37^{\circ} \mathrm{C}$ for one hour, washed in Aldefluor buffer, and maintained in $4^{\circ} \mathrm{C}$ throughout the process of ALDH assay per the manufacturer's instructions. High ALDH activity was assessed using the FL1 channel of a BD FACSAria Cell Sorting System and FACSDiva software (version 6.1.2; Becton, Dickinson and Company, San Jose, CA). Collected cells were analyzed for high ALDH activity with fluorescence-activated cell sorting (FACS), according to their fluorescence intensity, which corresponds to their ALDH activity levels, as well as low side scatter $\left(\mathrm{SCC}^{\mathrm{lo}}\right)$.

\subsection{Disulfiram Treatment}

Cultured human tumor cells were trypsinized, washed in DPBS, and counted using a hemocytometer. Cells were then plated in a 12-well dish (10,000 cells/well, in $1 \mathrm{~mL}$ of PM). Cells were allowed to adhere to the flask overnight and treated with disulfiram at concentrations of $0,500 \mathrm{nM}, 1 \mathrm{uM}$, and $1.5 \mathrm{uM}$. 48 hours later, the cells were fixed with $4 \%$ paraformaldehyde for 10 mins. The brightfield images of the cells were taken using a Nikon Eclipse E800 microscope (Melville, NY) equipped with a Retiga Exi digital camera (QImaging). All images were acquired and analyzed using Northern Eclipse software (version 6.0; Empix Imaging).

\subsection{Doxorubicin Treatment}

Human bone sarcoma cells were evaluated for response to doxorubicin, a cytotoxic chemotherapy often used in the treatment of bone sarcomas. Cells were plated 50,000 cells/well in 12 well plates in proliferation medium. Cells were allowed to proliferate at $37 \mathrm{C}$ for 24 hours. Doxorubicin was then added in varying concentrations ( 0 , $25 \mathrm{uM}, 50 \mathrm{uM}, 100 \mathrm{uM}, 250 \mathrm{uM}$, and $500 \mathrm{uM}$ ) and the cells were incubated for 24 hours. At that point, cells were fixed in $10 \%$ formaldehyde for 5 minutes. The brightfield images of the cells were taken using a Nikon Eclipse E800 microscope (Melville, NY) equipped with a Retiga Exi digital camera (QImaging). All images were acquired and analyzed using Northern Eclipse software (version 6.0; Empix Imaging).

\subsection{Statistical Analysis}

A student's $t$ test was used to calculate a p value between metastatic and non-metastatic groups. A p value of less than 0.05 was considered the threshold for statistical significance. Statistical analyses were performed by a biostatistician not directly involved with the project.

\section{Results}

\subsection{Bone Sarcoma Patients and ALDH Activity}

Table 1 illustrates the age, gender, histologic diagnosis, site of metastasis (where applicable), ALDH activity of each patient's cultured tumor cells, and standard deviations of the ALDH values when more than one measurement was performed. There were seven males and three females and the average age of the entire cohort was 57.4 years at the time of diagnosis. There were three cases of OS, five cases of CSA, and two cases of ES. The average age of the five patients with OS and EWS was 45 years, and the average age of the five CSA patients was 69.8 years. Of the patients who experienced metastases, $87.5 \%$ (seven of eight) experienced pulmonary metastases. Two patients experienced metastases to additional locations besides the lung (brain, bone) and one patient developed lymph node metastases. The average percentage of ALDH-high cells among the patients who had metastases was 18.1, and 2.1 in non-metastatic patients, with standard deviations of 12.6 and 1.3, respectively. According to our statistician, the difference in the percentage of ALDH-high cells between the metastatic and non-metastatic patients did reach statistical significance with a p value of 0.0091. If ALDH-high percentage of 3.1 was used as a threshold value, high ALDH activity correlated $100 \%$ with the incidence of metastases.

Figure 1 illustrates a sample ALDH activity assay from patient \#4, a 63-year-old female with CSA metastatic to her lungs. $20.3 \%$ of her cells displayed high ALDH activity. Figure 2 displays the ALDH activity of patient \#3, an 86 years old male with non-metastatic CSA. Note the difference in the percentage of ALDH-high cells, as depicted by the green curves. 
Table 1. Summarizes our series of ten consecutive patients with bone sarcomas.

\begin{tabular}{cccccc}
\hline Patient age & Gener (M/F) & Histology (OS,CSA,ES) & Site of mets & ALDH & ALDH st dev \\
\hline 70 & M & CSA & Lung & 23.2 & 2.7 \\
80 & M & CSA & - & 3.05 & 2.85 \\
86 & M & CSA & - & 1.2 & - \\
63 & F & CSA & Lung & 20.3 & - \\
19 & M & OS & Lymph node & 39.4 & - \\
35 & M & ES & Lung, brian & 4.5 & 2.6 \\
27 & M & ES & Lung, bone & 26.6 & - \\
50 & M & CSA & Lung & 21.2 & - \\
60 & F & OS & Lung & 3.9 & - \\
84 & F & OS & Lung & 5.5 & - \\
\hline
\end{tabular}

\begin{tabular}{|c|c|c|c|c|c|c|c|}
\hline 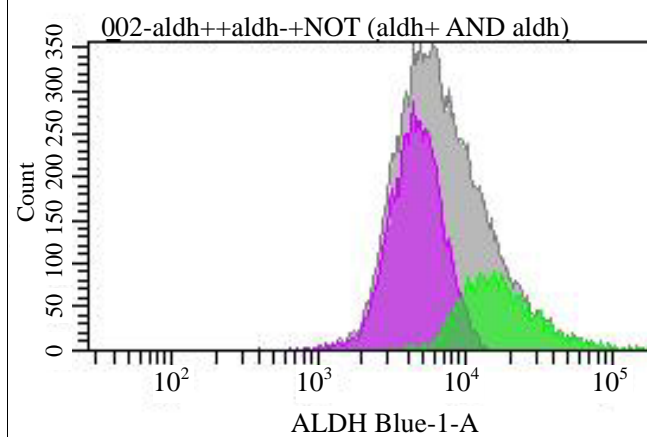 & $\begin{array}{l}\text { Specimen Name: } \\
\text { Tube Name: } \\
\text { SAMPLE ID: } \\
\text { DEAB?: } \\
\text { Population } \\
\square \text { Live Cells } \\
\text { fsc-s } \\
\square \text { aldh+ } \\
\text { aldh- } \\
\square \text { NOT (aldh+ A }\end{array}$ & $\begin{array}{l}\text { Tumors } \\
-002 \\
1 \\
\text { NO } \\
\\
\\
\\
\text { ND aldh- }\end{array}$ & $\begin{array}{c}\text { \#Events } \\
\# \# \# \text { } \\
\# \# \# \# \\
3.086 \\
7.260 \\
-) 15.179\end{array}$ & $\begin{array}{c}\text { \%Parent } \\
\# \# \# \text { } \\
\# \# \# \# \\
20.3 \\
47.8 \\
100.0\end{array}$ & $\begin{array}{c}\text { \% Gran... } \\
\# \# \# \# \\
56.8 \\
15.4 \\
36.1 \\
75.5\end{array}$ & $\begin{array}{c}\text { ALDH... } \\
\text { Mean } \\
11.404 \\
9.578 \\
20.734 \\
4.977 \\
9.578\end{array}$ & $\begin{array}{c}\begin{array}{c}\mathrm{LDH} . . . \\
\text { \%rCV }\end{array} \\
66.0 \\
61.1 \\
51.8 \\
38.7 \\
61.1\end{array}$ \\
\hline
\end{tabular}

Figure 1. Demonstrates the ALDH activity of patient \#4, a 63 years old female with CSA metastatic to her lungs. Her percentage of ALDH-high cells was 20.3. The average of percentage of ALDH-high cells in patients with metastatic disease was 18.1 (standard deviation $=12.6$ ).

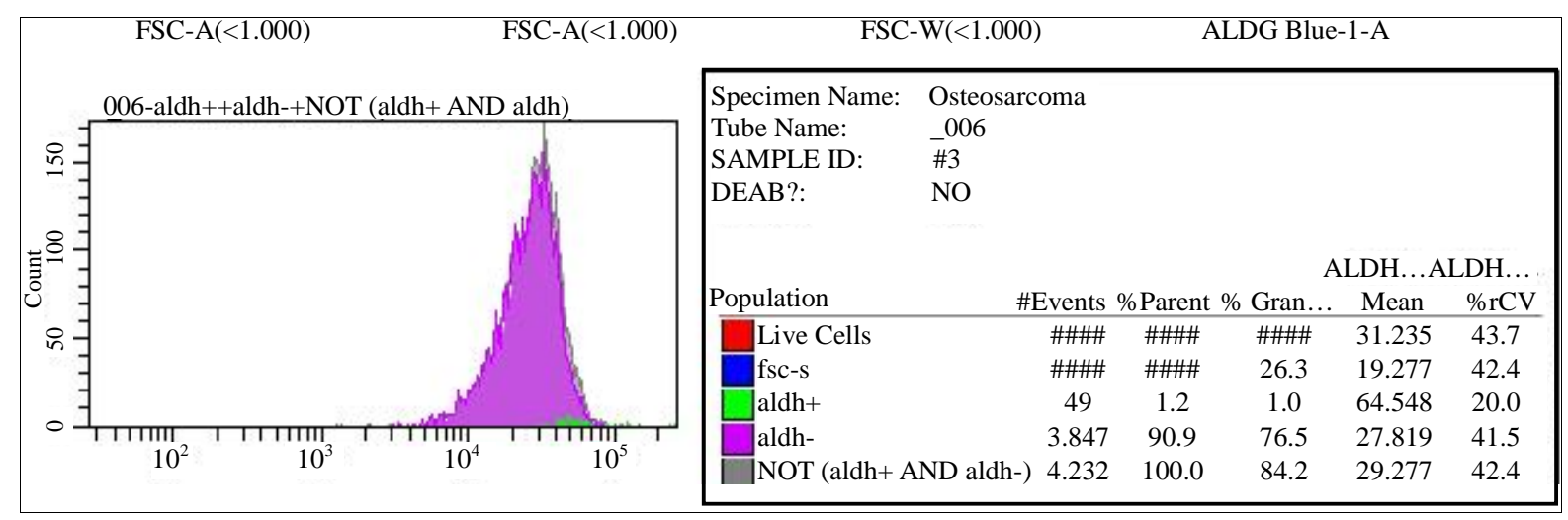

Figure 2. Depicts the ALDH activity of patient \#2, an 86 years old male with non-metastatic CSA. His percentage of ALDH-high cells was 1.2. The average percentage of ALDH-high cells in non-metastatic patients was 2.1 (standard deviation =1.3).

\subsection{Disulfiram and Doxorubicin Experiments}

Both disulfiram and doxorubicin decreased bone sarcoma cell proliferation in a dose-dependent fashion (Figures 3-5). 
Patient\#5, 19M with OS metastic to lymph node

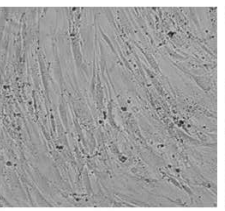

control

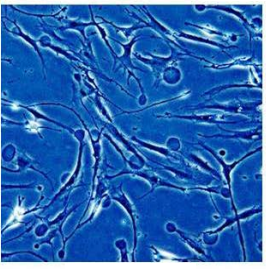

control

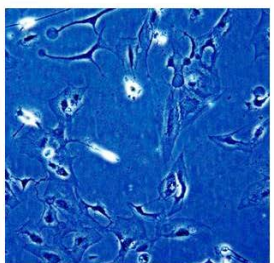

Dox 100 uM

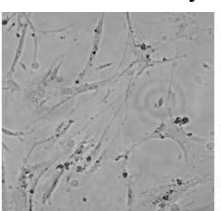

Disulfiram
500nM

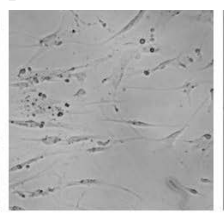

Disulfiram $1 \mathrm{uM}$

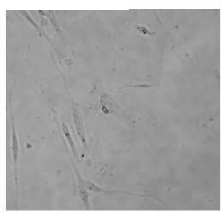

Disulfiram

$1.5 \mathrm{uM}$

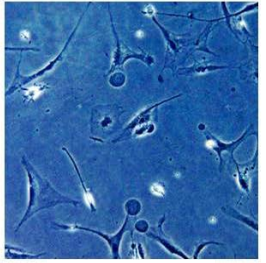

Dox 25 uM

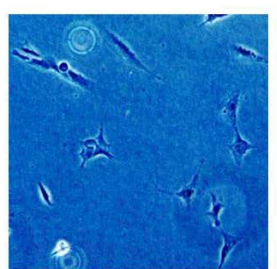

Dox 250 uM

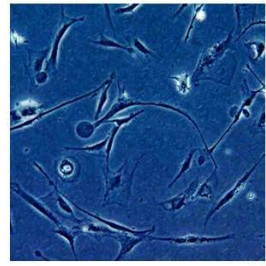

Dox 50 uM

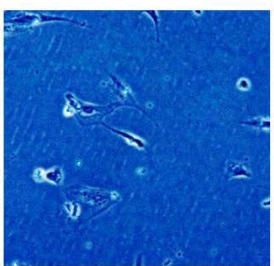

Dox 500 uM

Figure 3. Demonstrates the response to disulfiram and doxorubicin (Dox) in patient \#5, a 19 years old male with OS metastatic to his lymph nodes. Both disulfiram and doxorubicin demonstrated qualitative decreases in cell proliferation).

Patient\#2, 80M with non-metastatic CSA

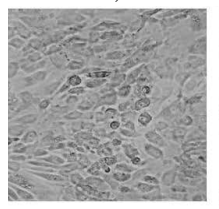

control

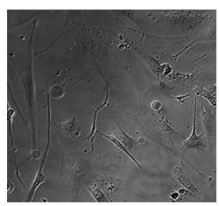

Disulfiram

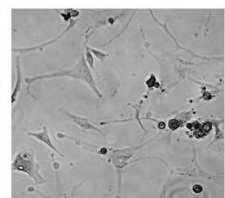

Disulfiram

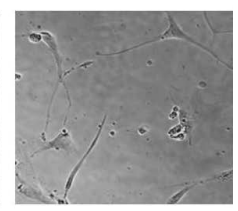

Disulfiram

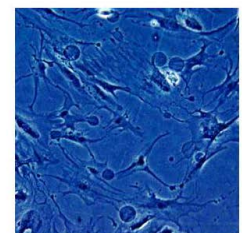

control

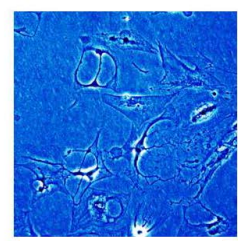

Dox $100 \mathrm{uM}$

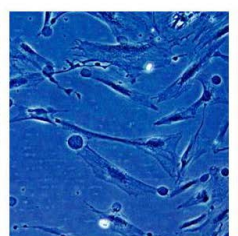

Dox 25 uM

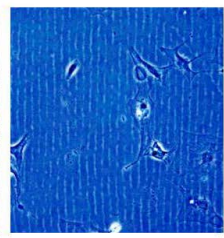

Dox 250 uM

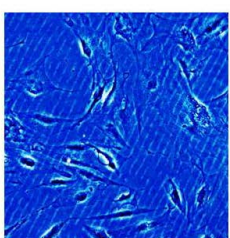

Dox $50 \mathrm{uM}$

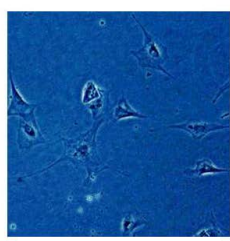

Dox 500 uM

Figure 4. Shows the responses to disulfiram and doxorubicin (Dox) of patient \#2, an 80 years old male with non-metastatic CSA. Both disulfiram and doxorubicin demonstrated qualitative decreases in cell proliferation. 


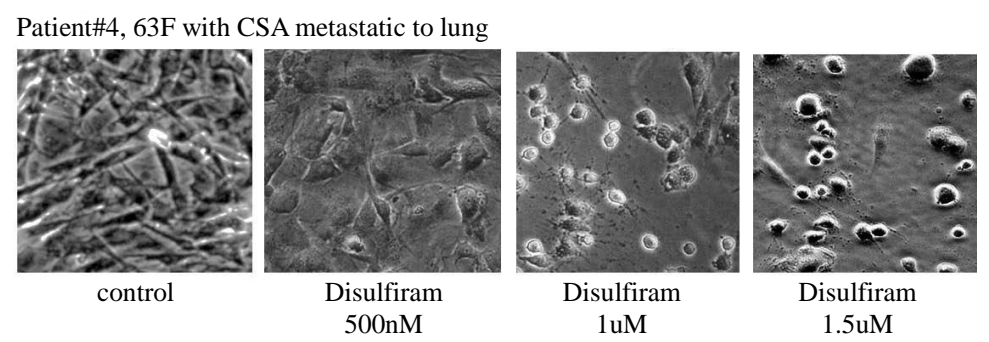

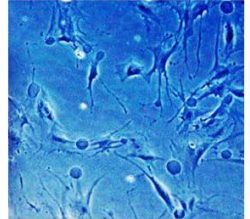

control

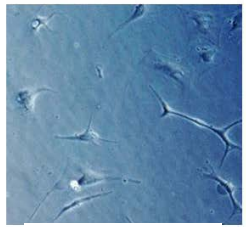

Dox $100 \mathrm{uM}$

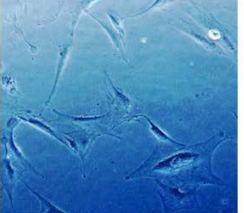

Dox 25 uM

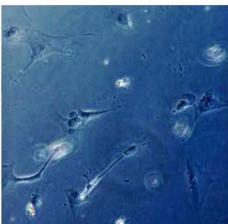

Dox $250 \mathrm{uM}$

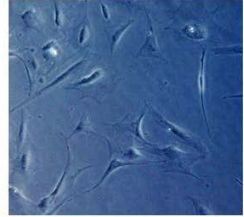

Dox $50 \mathrm{uM}$

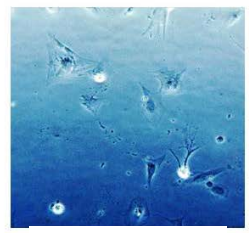

Dox 500 uM

Figure 5. Displays the histology of patient \#4, a 63 years old female with CSA metastatic to her lungs. Both disulfiram and doxorubicin (Dox) demonstrated qualitative decreases in cell proliferation.

\section{Discussion}

Bone sarcomas are rare, primary skeletal neoplasia. The most common forms are OS, CSA, and ES. Like all sarcomas, the prognosis of these diseases is ultimately determined by the presence of metastatic disease [9]-[12]. Others have demonstrated that ALDH expression and activity correlates with prognosis in other neoplasia, but this relationship has not been thoroughly evaluated in sarcomas of bone. We have previously demonstrated in murine OS cells that high ALDH activity correlates with murine OS cell biology [15] [16]. Here we report on the level of ALDH activity in a consecutive series of bone sarcoma patients.

This study has several limitations, not the least of which is the small series $(n=10)$ of bone sarcoma patients. While we did demonstrate a statistically significant difference $(\mathrm{p}=0.0091)$ between the percentage of ALDHhigh cells in metastatic versus non-metastatic patients, we are reluctant to draw any broad conclusions at this time. These data, while novel and encouraging, are very limited. Greater numbers of both metastatic and nonmetastatic bone sarcoma patients should be studied in order to confirm or refute the hypotheses that ALDH activity correlates with bone sarcoma metastatic potential, and that bone sarcoma cell metastatic potential can be affected via ALDH inhibition.

As with our murine OS cell data, we appreciated a dose-dependent effect with disulfiram treatment. While this was not completely surprising in our human OS patient, it was interesting to observe this in the CSA patients as well. CSA is remarkably chemo- and radio-resistant. CSA is therefore treated with surgery alone, as other adjuvants do not impact upon survival. We hypothesize that the ability to withstand oxidative stress via high ALDH activity is an essential attribute that sarcoma cells must possess in order to metastasize throughout the body. The feasibility of disulfiram or other ALDH inhibitors as much-needed adjuvants in the treatment of CSA and other bone sarcomas must be investigated, and these experiments are under way. Additionally, the importance of ALDH activity vis-a-vis the metastatic potential of soft tissue sarcoma should also be evaluated with a similar strategy outlined above.

\section{Conclusion}

In conclusion, we have demonstrated a significant correlation between ALDH activity and metastatic potential 
in a consecutive series of ten patients with OS, CSA, and ES. We have also demonstrated that human OS and CSA cells are sensitive to ALDH inhibition with disulfiram, which raises the possibility of using ALDH inhibition as a therapeutic strategy in bone sarcomas. These data are fascinating, but additional investigations are required to understand more completely the relationship between ALDH and metastatic potential in bone sarcomas.

\section{Acknowledgements}

The authors wish to thank David Biau MD, PhD for his assistance with the statistical analysis. This work was supported by NIH grant 1K08CA177927-01, the Shadyside Hospital Foundation, PittsburghCureSarcoma, the Pittsburgh Foundation, and the Houy family in loving memory of Jonathan Houy.

\section{References}

[1] Dai, X., Ma, W., He, X. and Jha, R.K. (2011) Review of Therapeutic Strategies for Osteosarcoma, Chondrosarcoma, and Ewing's Sarcoma. Medical Science Monitor, 17, RA177. http://dx.doi.org/10.12659/MSM.881893

[2] Damron, T.A., Ward, W.G. and Stewart, A. (2007) Osteosarcoma, Chondrosarcoma, and Ewing's Sarcoma: National Cancer Data Base Report. Clinical Orthopaedics and Related Research, 459, 40-47. http://dx.doi.org/10.1097/BLO.0b013e318059b8c9

[3] Hameed, M. and Dorfman, H. (2011) Primary Malignant Bone Tumors-Recent Developments. Seminars in Diagnostic Pathology, 28, 86-101. http://dx.doi.org/10.1053/j.semdp.2011.02.002

[4] Qureshi, A., Ahmad, Z., Azam, M. and Idrees, R. (2010) Epidemiological Data for Common Bone Sarcomas. Asian Pacific Journal of Cancer Prevention, 11, 393-395.

[5] Skubitz, K.M. and D’Adamo, D.R. (2007) Sarcoma. Mayo Clinic Proceedings, 82, 1409-1432. http://dx.doi.org/10.4065/82.11.1409

[6] Whelan, J., McTiernan, A., Cooper, N., Wong, Y.K., Francis, M., Vernon, S., et al. (2012) Incidence and Survival of Malignant Bone Sarcomas in England 1979-2007. International Journal of Cancer, 131, E508-E517. http://dx.doi.org/10.1002/ijc.26426

[7] Brage, M.E. and Simon, M.A. (1992) Evaluation, Prognosis, and Medical Treatment Considerations of Metastatic Bone Tumors. Orthopedics, 15, 589-596.

[8] Riccio, A.I., Wodajo, F.M. and Malawer, M. (2007) Metastatic Carcinoma of the Long Bones. American Family Physician, 76, 1489-1494.

[9] Chmielowski, B., Federman, N. and Tap, W.D. (2012) Clinical Trial End Points for Assessing Efficacy of Novel Therapies for Soft-Tissue Sarcomas. Expert Review of Anticancer Therapy, 12, 1217-1228. http://dx.doi.org/10.1586/era.12.100

[10] Kondo, T., Suehara, Y., Kikuta, K., Kubota, D., Tajima, T., Mukaihara, K., et al. (2013) Proteomic Approach toward Personalized Sarcoma Treatment: Lessons from Prognostic Biomarker Discovery in Gastrointestinal Stromal Tumor. Proteomics-Clinical Applications, 7, 70-78. http://dx.doi.org/10.1002/prca.201200085

[11] Schoffski, P. (2012) Pazopanib in the Treatment of Soft Tissue Sarcoma. Expert Review of Anticancer Therapy, 12, 711-723. http://dx.doi.org/10.1586/era.12.41

[12] Sinha, S. and Peach, A.H. (2010) Diagnosis and Management of Soft Tissue Sarcoma. BMJ, 341, c7170. http://dx.doi.org/10.1136/bmj.c7170

[13] Guma, S.R., Lee, D.A., Yu, L., Gordon, N., Hughes, D., Stewart, J., et al. (2014) Natural Killer Cell Therapy and Aerosol Interleukin-2 for the Treatment of Osteosarcoma Lung Metastasis. Pediatric Blood \& Cancer, 61, 618-626. http://dx.doi.org/10.1002/pbc.24801

[14] Heck Jr., R.K., Peabody, T.D. and Simon, M.A. (2006) Staging of Primary Malignancies of Bone. CA: A Cancer Journal for Clinicians, 56, 366-375. http://dx.doi.org/10.3322/canjclin.56.6.366

[15] Mu, X., Isaac, C., Greco, N., Huard, J. and Weiss, K. (2013) Notch Signaling Is Associated with ALDH Activity and an Aggressive Metastatic Phenotype in Murine Osteosarcoma Cells. Frontiers in Oncology, 3, 143. http://dx.doi.org/10.3389/fonc.2013.00143

[16] Mu, X., Isaac, C., Schott, T., Huard, J. and Weiss, K. (2013) Rapamycin Inhibits ALDH Activity, Resistance to Oxidative Stress, and Metastatic Potential in Murine Osteosarcoma Cells. Sarcoma, 2013, 480713. http://dx.doi.org/10.1155/2013/480713

[17] Mu, X., Sultankulov, B., Agarwal, R., Mahjoub, A., Schott, T., Greco, N., et al. (2013) Chick Embryo Extract Demethylates Tumor Suppressor Genes in Osteosarcoma Cells. Clinical Orthopaedics and Related Research, 472, 865-873. 
http://dx.doi.org/10.1007/s11999-013-3104-6

[18] Riad, S., Griffin, A.M., Liberman, B., Blackstein, M.E., Catton, C.N., Kandel, R.A., et al. (2004) Lymph Node Metastasis in Soft Tissue Sarcoma in an Extremity. Clinical Orthopaedics and Related Research, 426, 129-134. http://dx.doi.org/10.1097/01.blo.0000141660.05125.46

[19] Herget, G.W., Uhl, M., Opitz, O.G., Adler, C.P., Sudkamp, N.P. and Knoller, S. (2011) The Many Faces of Chondrosarcoma of Bone, Own Cases and Review of the Literature with an Emphasis on Radiology, Pathology and Treatment. Acta chirurgiae orthopaedicae et traumatologiae Cechoslovaca, 78, 501-509.

[20] Mavrogenis, A.F., Ruggieri, P., Mercuri, M. and Papagelopoulos, P.J. (2011) Dedifferentiated Chondrosarcoma Revisited. Journal of Surgical Orthopaedic Advances, 20, 106-111.

[21] Gordon, N. and Kleinerman, E.S. (2010) Aerosol Therapy for the Treatment of Osteosarcoma Lung Metastases: Targeting the Fas/FasL Pathway and Rationale for the Use of Gemcitabine. Journal of Aerosol Medicine and Pulmonary Drug Delivery, 23, 189-196. http://dx.doi.org/10.1089/jamp.2009.0812

[22] Weiss, K.R., Cooper, G.M., Jadlowiec, J.A., McGough III, R.L. and Huard, J. (2006) VEGF and BMP Expression in Mouse Osteosarcoma Cells. Clinical Orthopaedics and Related Research, 450, 111-117. http://dx.doi.org/10.1097/01.blo.0000229333.98781.56

[23] Bortolomai, I., Canevari, S., Facetti, I., De Cecco, L., Castellano, G., Zacchetti, A., et al. (2010) Tumor Initiating Cells: Development and Critical Characterization of a Model Derived from the A431 Carcinoma Cell Line Forming Spheres in Suspension. Cell Cycle, 9, 1194-1206. http://dx.doi.org/10.4161/cc.9.6.11108

[24] Burger, P.E., Gupta, R., Xiong, X., Ontiveros, C.S., Salm, S.N., Moscatelli, D., et al. (2009) High Aldehyde Dehydrogenase Activity: A Novel Functional Marker of Murine Prostate Stem/Progenitor Cells. Stem Cells, 27, $2220-2228$. http://dx.doi.org/10.1002/stem.135

[25] Charafe-Jauffret, E., Ginestier, C., Iovino, F., Tarpin, C., Diebel, M., Esterni, B., et al. (2010) Aldehyde Dehydrogenase 1-Positive Cancer Stem Cells Mediate Metastasis and Poor Clinical Outcome in Inflammatory Breast Cancer. Clinical Cancer Research, 16, 45-55. http://dx.doi.org/10.1158/1078-0432.CCR-09-1630

[26] Clay, M.R., Tabor, M., Owen, J.H., Carey, T.E., Bradford, C.R., Wolf, G.T., et al. (2010) Single-Marker Identification of Head and Neck Squamous Cell Carcinoma Cancer Stem Cells with Aldehyde Dehydrogenase. Head Neck, 32, 11951201. http://dx.doi.org/10.1002/hed.21315

[27] Croker, A.K., Goodale, D., Chu, J., Postenka, C., Hedley, B.D., Hess, D.A., et al. (2009) High Aldehyde Dehydrogenase and Expression of Cancer Stem Cell Markers Selects for Breast Cancer Cells with Enhanced Malignant and Metastatic Ability. Journal of Cellular and Molecular Medicine, 13, 2236-2252. http://dx.doi.org/10.1111/j.1582-4934.2008.00455.X

[28] Deng, S., Yang, X., Lassus, H., Liang, S., Kaur, S., Ye, Q., et al. (2010) Distinct Expression Levels and Patterns of Stem Cell Marker, Aldehyde Dehydrogenase Isoform 1 (ALDH1), in Human Epithelial Cancers. PLoS One, 5, e10277. http://dx.doi.org/10.1371/journal.pone.0010277

[29] Ginestier, C., Hur, M.H., Charafe-Jauffret, E., Monville, F., Dutcher, J., Brown, M., et al. (2007) ALDH1 Is a Marker of Normal and Malignant Human Mammary Stem Cells and a Predictor of Poor Clinical Outcome. Cell Stem Cell, 1, 555-567. http://dx.doi.org/10.1016/j.stem.2007.08.014 